\title{
Variación anual de larvas del mejillón dorado (Limnoperna fortunei) en sistemas de refrigeración de centrales hidroeléctricas en embalses del Río Negro, Uruguay
}

Annual variation of golden mussel larvae (Limnoperna fortunei) in refrigeration systems of hydroelectric power plants in Río Negro reservoirs, Uruguay

Variação anual das larvas do mexilhão dourado (Limnoperna fortunei) nos sistemas de refrigeração de usinas hidrelétricas em reservatorios do Río Negro, Uruguai

Daniel Fabián ${ }^{1 *}$ https://orcid.org/0000-0001-6108-1949

Carolina Ferrer ${ }^{1}$ https://orcid.org/0000-0003-0133-243X

Jennifer Pereira ${ }^{1}$ https://orcid.org/0000-0001-5170-8250

Pablo Muniz ${ }^{1}$ https://orcid.org/0000-0001-5310-3781

Leandro Capurro ${ }^{1}$ https://orcid.org/0000-0002-7651-4337

Magdalena Mandiá ${ }^{2}$ https://orcid.org/0000-0002-0262-9422

Guillermo Failache ${ }^{2}$ https://orcid.org/0000-0003-4174-4797

Ernesto Brugnoli ${ }^{1}$ https://orcid.org/0000-0001-7304-1856

*Autor de contacto: danielfabianroland@gmail.com

${ }^{1}$ Oceanografía y Ecología Marina, Facultad de Ciencias, Universidad de la República, Montevideo, Uruguay

${ }^{2}$ Gerencia Generación Hidráulica y Eólica, UTE, Montevideo, Uruguay

Recepción: 6 Setiembre 2020 Aprobación: 21 Mayo 2021

Esta obra está bajo una Licencia Creative Commons Atribución-NoComercial 4.0 Internacional. 
Resumen: Limnoperna fortunei (mejillón dorado) es un molusco invasor de la cuenca del Plata, originario del sureste asiático. Las colonias bentónicas generan macrofouling en instalaciones hidráulicas, ocasionando gastos en las empresas afectadas. El objetivo del trabajo es determinar la variación de la densidad y el tamaño de los estadios larvales valvados del mejillón dorado, estableciendo asociaciones con los parámetros de calidad de agua en sistemas de refrigeración de las tres centrales hidroeléctricas $(\mathrm{CH})$ de los embalses del Río Negro (Uruguay) invadidos por la especie. En los tres sistemas de refrigeración, la densidad larval promedio del mejillón dorado presentó asociaciones con la temperatura del agua y el porcentaje de materia orgánica en agua. Se registraron las mayores densidades larvales durante los meses cálidos, y las mínimas durante los más fríos. Para los tres sistemas se sugiere un descanso reproductivo de la especie a valores menores a $18^{\circ} \mathrm{C}$ de temperatura del agua e inicio del ciclo larval a valores superiores. Los estadios valvados de larva Velíger tipo D y Velíger preumbonada fueron los estadios dominantes. El sistema de refrigeración de $\mathrm{CH}$ Constitución fue el más expuesto al asentamiento del mejillón dorado. Se destaca la necesidad de continuar profundizando en los estudios de $L$. fortunei para implementar medidas de control y mitigación de la especie.

Palabras clave: especie invasora acuática, incrustación biológica, molusco, manejo.

Abstract: Limnoperna fortunei (golden mussel) is an alien mollusk in the Plata basin and is native from the Southeastern Asia. Its benthic patches generate macrofouling in hydraulic installations causing additional costs to the affected companies. The objective of this work is to determine the variation of abundance and the size of the valves larval stages of the golden mussel, establishing associations with the water quality parameters in the cooling systems in the three hydroelectric power stations (HP) of the reservoirs of the Negro River (Uruguay) that has been invaded by the species. In the three cooling systems the golden mussel larval density average showed associations with temperature of the water and the percentage of organic material of the column water. Larger larval abundances were registered during warm and minimum during cold months. For the three systems, is suggested a reproductive pause of the species at water temperatures lower than $18^{\circ} \mathrm{C}$ and the start of the larval cycle at higher values. The Velíger larvae type D and preumbonate Veliger stages were the dominant stages. In terms of the golden mussel settlement the HP Constitución cooling system was the most exposed. It highlights the need to continue deepening in the knowledge of $L$. fortunei in order to better implement control and mitigation measures for the species.

Keywords: invasive aquatic species, macrofouling, mollusk, management.

Resumo: Limnoperna fortunei (mexilhão dourado) é um molusco invasor da bacia do Prata, originário do sudeste asiático. Suas colônias bentônicas geram macro incrustações (macrofouling) nas instalações hidráulicas gerando gastos adicionais nas empresas afetadas. O objetivo deste trabalho foi determinar a variação da abundância e tamanho dos estádios larvais valvados do mexilhão dourado, estabelecendo possíveis associações com variáveis de qualidade da água nos sistemas de refrigeração em três usinas hidrelétricas $(\mathrm{CH})$ dos reservatórios invadidos do Río Negro (Uruguai). Nos três sistemas de refrigeração as médias dos estádios larvais do mexilhão dourado apresentaram associações com a temperatura da água e a porcentagem de matéria orgânica. Foram registradas maiores abundâncias larvais nos meses mais cálidos e menores nos mais frios. Para os três sistemas, sugere-se um repouso 
reprodutivo da espécie em valores inferiores a $18^{\circ} \mathrm{C}$ de temperatura da água e o início do ciclo larval em valores superiores. Os estádios valvados de larva Velíger tipo D e Velíger préumbonada fôramos dominantes. Em termos de assentamento de mexilhões o sistema $\mathrm{CH}$ Constitución foi o mais exposto. Destaca-se a necessidade de continuar gerando conhecimento sobre essa espécie invasorade forma tal que as medidas que se adotem para programar o controle e a mitigação da espécie tenham maior sustento científico-técnico.

Palavras-chave: espécie invasora aquâtica, incrustação biológica, molusco, gestão.

\section{INTRODUCCIÓN}

Limnoperna fortunei (Dunker 1857), conocido como mejillón dorado, es una especie de molusco invasor de la cuenca del Plata, originaria de sistemas de agua dulce del sudeste asiático (Darrigran, 2002). En Uruguay se registró por primera vez en 1994 en zonas costeras del Río de la Plata (Scarabino y Verde, 1995), presentando actualmente una amplia distribución en las cuencas del Río de la Plata, Río Negro, Río Uruguay, Río Santa Lucía, Laguna Merín (Brugnoli, et al., 2005; Langone, 2005) y más recientemente en Laguna del Sauce (Clemente, et al., 2015). Desde su llegada a la región ha colonizado diversos tipos de sustratos consolidados (naturales y artificiales), y ha presentado un aumento en su abundancia poblacional y cambios en la composición de la comunidad bentónica y dietas de peces autóctonos de la cuenca del Plata. Adicionalmente, causa incrustaciones (macrofouling), ocasionando daños en instalaciones hidráulicas (Darrigran, et al., 1998; Darrigran y Ezcurra de Drago, 2000; Montalto, et al., 1999; Penchaszadeh, et al., 2000; Mansur, et al., 2003; Brugnoli, et al., 2005; Boltovskoy y Correa, 2015).

Durante su ciclo biológico, presenta diversos estadios larvales y posteriormente desarrolla estructuras para la fijación. La etapa de asentamiento ocurre en los últimos estadios larvales con organismos que presentan tamaños entre 500-800 um (Ezcurra de Drago, et al., 2006). Este tamaño de organismo no presenta barreras físicas en los diferentes sistemas de conducción hidráulica debido a que la mayoría de los sistemas de captación consideran sistemas de filtración o rejas con tamaños de poro mayores a $1000 \mu \mathrm{m}$. De esta manera, las larvas y estadios con estructuras de fijación (filamentos bisales) ingresan, se asientan y desarrollan colonias bentónicas en el interior de los sistemas de conducción hidráulica, o en diferentes estructuras humanas (Darrigran, 2002; Darrigran, et al., 2007; Boltovskoy, 2015). Las colonias bentónicas asentadas ocasionan macrofouling, afectando los servicios ecosistémicos de los recursos hídricos de la cuenca del Plata (Brugnoli, et al., 2006; Darrigran, et al., 2007; Boltovskoy y Correa 2015). Entre los servicios ecosistémicos afectados, destacan usos del agua con fines energéticos, potabilización, riego, refrigeración o para uso agroindustrial (Darrigran, 2002; Boltovskoy, 2015). El mejillón dorado ha ocasionado obstrucción de filtros, inutilización de sensores hidráulicos, daños en las bombas de captación y disminución del diámetro de tuberías de conducción del agua. Estos efectos generan gastos en 
plantas potabilizadoras de agua, centrales nucleares, hidroeléctricas, refinerías, siderúrgicas y plantas agroindustriales (acuicultura, forestal, alimenticia), debido a tareas de mantenimiento y modificaciones estructurales, así como en planes de manejo y control poblacional de la especie (Muniz, et al., 2005; Brugnoli, et al., 2006; Boltovskoy, 2015).

El ciclo larval de la especie es muy flexible dado que se adapta a amplias variaciones ambientales (Damborenea y Penchaszadeh, 2006). La temperatura es una característica ambiental importante, que se acompaña de otras, como el fotoperíodo y la abundancia de fitoplancton, las cuales en conjunto favorecen el desove (Haag, et al., 1992; Ram, et al.,1996; Darrigran, et al., 2007). Entre los parámetros ambientales involucrados en su etapa larval, la densidad de los estadios larvales está relacionada con la temperatura del agua, dado que la actividad reproductiva de $L$. fortunei se ve interrumpida con valores menores a $16-17^{\circ} \mathrm{C}$ (Cataldo y Boltovskoy, 2000).

En el Río Negro se ubican tres embalses en cadena destinados mayormente a la generación de energía hidroeléctrica (Conde y Sommaruga, 1999). Estos ambientes se encuentran en una zona templada, apropiada para las larvas del mejillón dorado, donde la temperatura, $\mathrm{pH}$ y conductividad se encuentran dentro del rango adecuado de sobrevivencia de la especie (Darrigran y Damborenea, 2005; Darrigran, et al., 2007; Chalar, et al., 2012; Chalar, et al., 2015). En este sistema de embalses se registra la presencia de larvas y estadios bentónicos de L. fortunei desde 1999, mostrando un avance de la invasión en dirección río arriba (embalse Palmar hacia embalse del Rincón del Bonete) (Brugnoli, et al., 2005; Brugnoli, et al., 2011). Esta especie invadió los cuerpos de agua y el interior de las centrales hidroeléctricas, colonizando los sistemas de refrigeración de las turbinas, sensores hidráulicos, tuberías, cámaras y estructuras sumergidas (Clemente y Brugnoli, 2002; Brugnoli, et al., 2011).

El objetivo del presente trabajo es describir la variación anual (2018-2019) en la densidad y el tamaño de los estadios larvales valvados de $L$. fortunei y su relación con variables fisicoquímicas del agua en los sistemas de refrigeración de tres centrales hidroeléctricas (Rincón del Bonete, Baygorria y Constitución), generando recomendaciones de manejo para mitigar la problemática del mejillón dorado en las mencionadas $\mathrm{CH}$ del Río Negro.

\section{MATERIALES Y MÉTODOS}

\section{Área de estudio}

El Río Negro es el principal río interior de Uruguay, presenta una cuenca de 72000 $\mathrm{km}^{2}$, de los cuales $68875 \mathrm{~km}^{2}$ se encuentran en territorio uruguayo. Este río presenta un sistema de embalses (E-W): Rincón del Bonete $\left(1070 \mathrm{~km}^{2}\right)$, Baygorria $\left(100 \mathrm{~km}^{2}\right)$ y Palmar $\left(320 \mathrm{~km}^{2}\right)$, destinados a la producción de energía eléctrica, con una capacidad 
de generación de 600 MW (Conde y Sommaruga, 1999) (Figura 1). La CH Rincón del Bonete (situada en el embalse Rincón del Bonete) fue instalada en 1948, con una potencia de $155 \mathrm{MW}$ (4 turbinas); la $\mathrm{CH}$ Baygorria fue puesta en funcionamiento en 1960 y presenta 108 MW (3 turbinas), mientras que la CH Constitución (embalse Palmar) comenzó a funcionar en 1982, con una potencia instalada de 333 MW (3 turbinas).
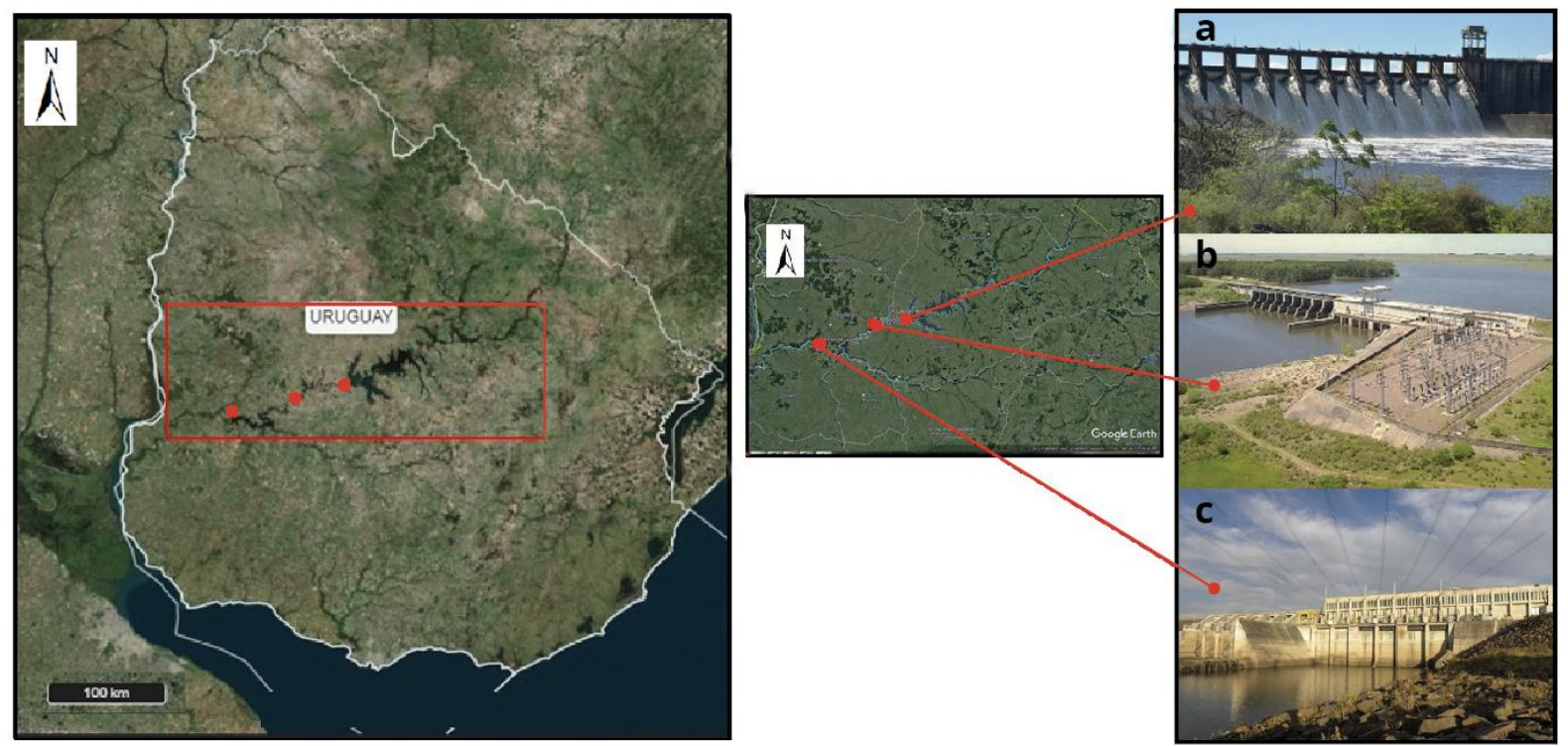

Figura 1. Área de estudio. a) $\mathrm{CH}$ Rincón del Bonete, b) $\mathrm{CH}$ Baygorria y c) $\mathrm{CH}$ Constitución.

\section{Estrategia de muestreo}

En los tres sistemas de refrigeración (SR) de las $\mathrm{CH}$ Rincón del Bonete, Baygorria y Constitución se escogió una turbina por $\mathrm{CH}$ para su monitoreo. La colecta de muestras de agua en los SR se realizó por técnicos de UTE previamente capacitados entre febrero de 2018 y febrero de 2019. Presentó una periodicidad de colecta quincenal durante los meses de primavera, verano e inicios de otoño (setiembre-abril), y mensual en otoño e invierno (mayo-agosto). Las muestras cuantitativas (larvas de L. fortunei) se colectaron por triplicado en los diferentes SR, filtrando $100 \mathrm{~L}$ de agua con una malla de $100 \mu \mathrm{m}$ y se fijaron in situ en alcohol (95\%). Complementariamente, en un punto de los SR se colectaron $5 \mathrm{~L}$ de agua para la determinación de variables fisicoquímicas $(\mathrm{pH}$, conductividad, sólidos suspendidos totales, materia orgánica en suspensión y \% materia orgánica). Las muestras fueron enviadas a Montevideo para su análisis en los laboratorios de Oceanografía y Ecología Marina (Facultad de Ciencias, UdelaR). Los registros continuos de temperatura del agua se obtuvieron a partir de sensores automáticos ubicados al ingreso de los SR y fueron facilitados por las gerencias de las diferentes $\mathrm{CH}$. 


\section{Análisis de laboratorio}

Una vez arribadas las muestras al laboratorio (24 horas de colectadas), se determinó la conductividad mediante un multiparámetro YSI 63, y el $\mathrm{pH}$ mediante $\mathrm{pH}$-metro EcoTester pH2. Para las concentraciones de sólidos totales en suspensión (SST), materia orgánica en suspensión y porcentaje de materia orgánica ( $\mathrm{MO}$ y \%MO), se filtraron $1000 \mathrm{~mL}$ por muestra en un filtro de $0,45 \mu \mathrm{m}$, se secaron $\left(60^{\circ} \mathrm{C}\right)$ y muflaron $\left(500^{\circ} \mathrm{C}\right)$, de acuerdo con Arocena (2016). La cuantificación de larvas de L. fortunei (densidad promedio total y densidad por estadio larval) se realizó en un microscopio óptico binocular a 10 y 40 aumentos, utilizando una cámara Sedwick-Rafter de $2 \mathrm{~mL}$, realizándose el conteo total de la muestra. La densidad se expresa en ind. $\mathrm{m}^{-3}$. Para la separación de los estadios se utilizó una serie de tamices de 1,2mm, 0,8mm, 0,5mm, $0,15 \mathrm{~mm}$ y $0,10 \mathrm{~mm}$ de apertura de malla. Los estadios valvares considerados se identificaron de acuerdo con Ezcurra de Drago y otros (2006) (Tabla 1).

Tabla 1. Características de los estadios larvales valvados analizados (modificado de Ezcurra de Drago, et al., 2006).

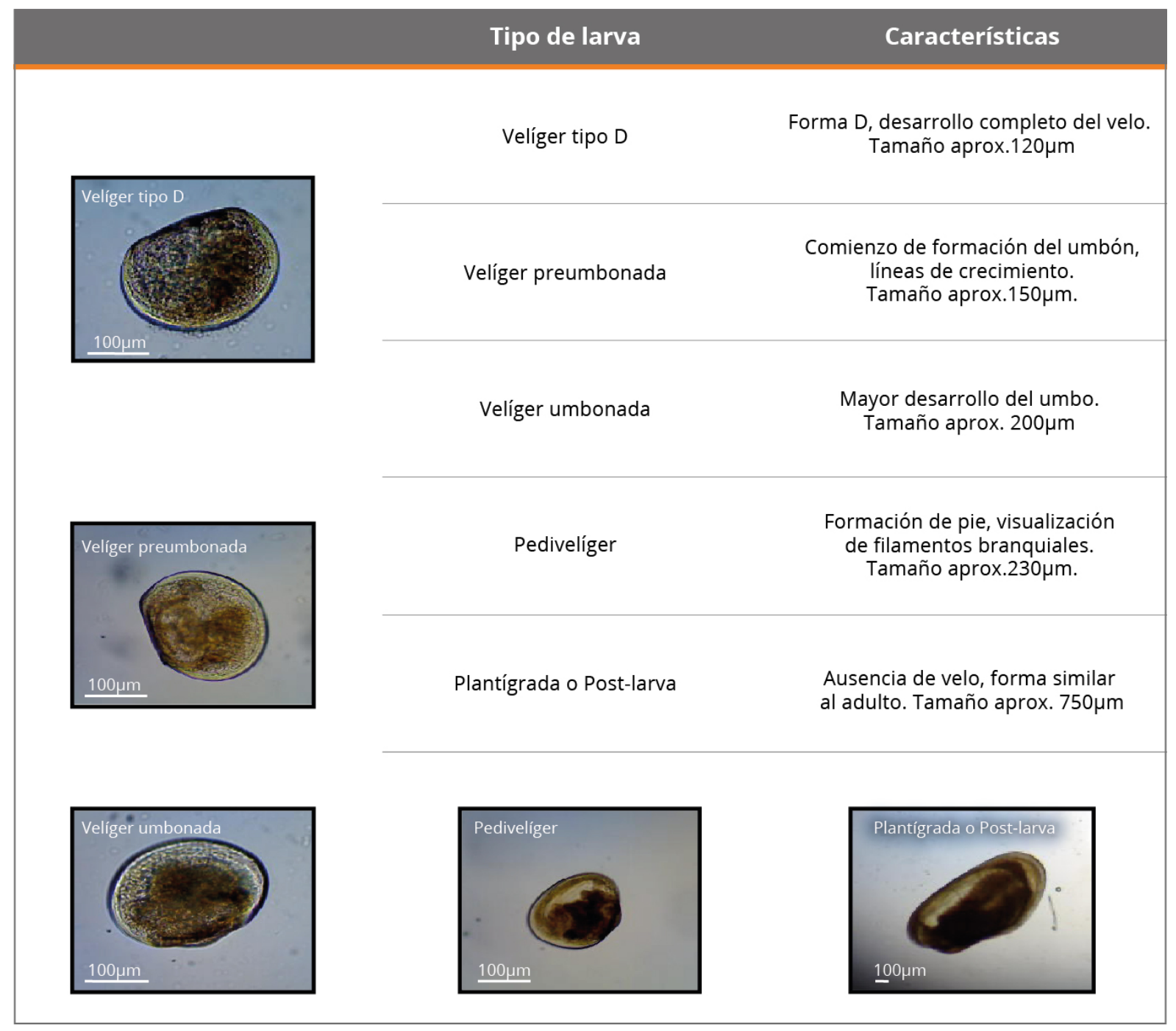




\section{Análisis de datos}

En las diferentes $\mathrm{CH}$ se determinaron los promedios y desvíos quincenales/mensuales de temperatura, conductividad, $\mathrm{pH}, \mathrm{SST}, \mathrm{MO}$ y \%MO. La conductividad $(K)$, los SST y el \%MO se transformaron mediante log $(x+1)$, según lo establecido por Reavie y otros (2010); para la densidad promedio larval se utilizó raíz cuarta (Xu, et al., 2011); mientras que el $\mathrm{pH}$ y la temperatura no fueron transformados. Se realizaron correlaciones de Pearson entre las variables fisicoquímicas y la densidad promedio larval, considerando un nivel de significancia del 99 y 95\%. Se realizó un Análisis de Componentes Principales (ACP) para identificar las asociaciones entre los parámetros ambientales y los promedios de densidades larvales, considerando las tres $\mathrm{CH}$ durante los diferentes muestreos. Para ambos análisis estadísticos no se consideró la MO. El ACP y las correlaciones se realizaron con el Software $R$ utilizando los paquetes ggfortify y ggplot2.

\section{RESULTADOS Y DISCUSIÓN}

La variación anual de la temperatura del agua en los sistemas de refrigeración para las tres $\mathrm{CH}$ presentó tendencias similares; valores máximos en verano (diciembrefebrero), una disminución a partir de marzo y valores mínimos en invierno (julioagosto) (Figura 2). A pesar de las tendencias similares, la $\mathrm{CH}$ Baygorria mostró períodos de temperatura mínimos y máximos más prolongados en el tiempo, en comparación con las CH Rincón del Bonete y Constitución.
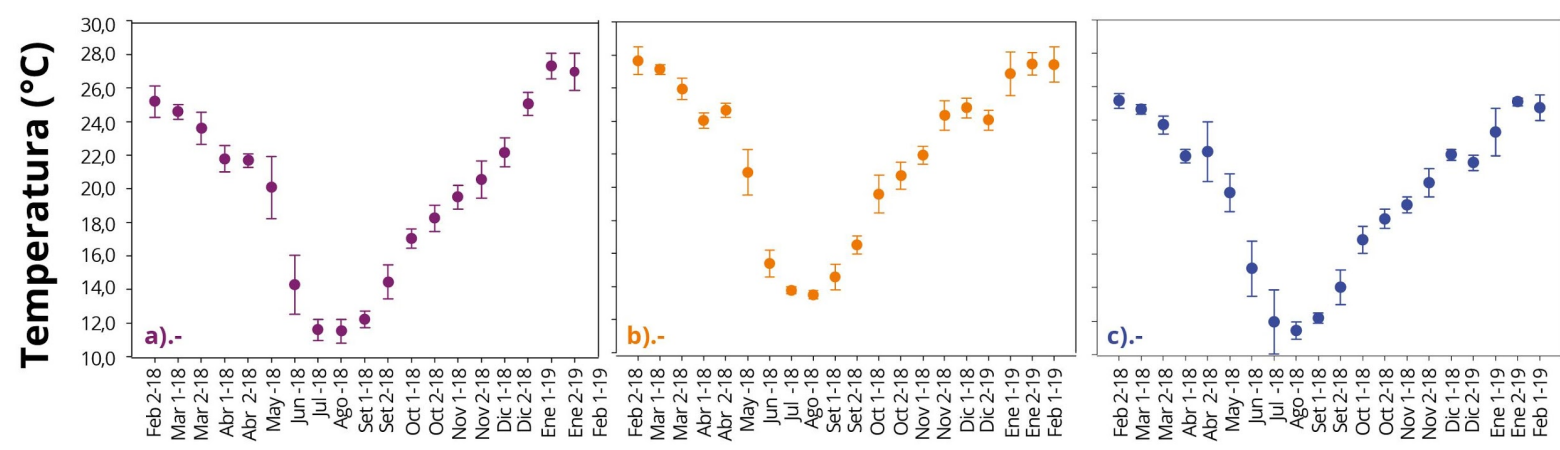

Figura 2. Promedios mensuales $(x \pm d s)$ de temperatura del agua en las tres $\mathrm{CH}: \mathrm{a}$ ) Rincón del Bonete b) Baygorria y c) Constitución, durante el período de estudio. Mes 1: primera quincena, Mes 2: segunda quincena; 18 y 19: años de colecta.

El pH presentó valores alcalinos en el $96 \%$ de los casos; los mínimos registrados fueron similares en las tres $\mathrm{CH}$ (6,84 Baygorria, 7,40 Rincón del Bonete y 6,81 Constitución). Los máximos se presentaron en Rincón del Bonete $(8,85)$, Baygorria 
$(8,90)$ y Constitución $(9,1)$. La conductividad osciló entre $64,4 \mu \mathrm{Scm}^{-1}$ y $203,1 \mu \mathrm{Scm}^{-1}$, los mínimos se observaron en la CH Rincón del Bonete (octubre 2018) y los máximos en la CH Baygorria (noviembre 2018). La mayor concentración de sólidos suspendidos totales (SST) se encontró en la CH Baygorria, en febrero $\left(18,8 \mathrm{mgL}^{-1}\right)$, y la concentración máxima de $\mathrm{MO}$ en la $\mathrm{CH}$ Rincón del Bonete, en abril $\left(5 \mathrm{mgL}^{-1}\right)$. Los \%MO mostraron un comportamiento similar en las tres $\mathrm{CH}$, con máximos durante verano $(\mathrm{CH}$ Baygorria, febrero) y mínimos durante el invierno (CH Constitución, julio) (Tabla 2). Las tendencias de los parámetros fisicoquímicos en los sistemas de refrigeración de las tres $\mathrm{CH}$ situadas en el Río Negro se ajustan con los encontrados en monitoreos de calidad de agua realizados en los embalses de dicho sistema fluvial (Chalar, et al., 2015).

Tabla 2. Variables fisicoquímicas del agua. Promedios (x), coeficiente de variación (CV), máximos y mínimos. Abreviaciones: K: conductividad, SST: sólidos suspendidos totales, MO: materia orgánica. Mes 1: primera quincena, Mes 2: segunda quincena; 18 y 19: años de colecta.

\begin{tabular}{|c|c|c|c|c|c|c|}
\hline & & $\mathrm{x} \pm \mathrm{CV}$ & Máximo & Mes & Mínimo & Mes \\
\hline \multirow{3}{*}{$\mathrm{pH}$} & Rincón del Bonete & $8,11 \pm 0,06$ & 8,85 & Abr 1-18 & 7,4 & Set 2-18 \\
\hline & Baygorria & $8,04 \pm 0,07$ & 8,9 & Dic 2-18 & 6,84 & Ene 2-19 \\
\hline & Constitución & $8,04 \pm 0,07$ & 9,1 & Ago 2-18 & 6,81 & Ene 2-19 \\
\hline \multirow{3}{*}{$\begin{array}{c}\mathrm{K} \\
\mu \mathrm{Scm}\end{array}$} & Rincón del Bonete & $84,70 \pm 0,32$ & 178,70 & May 2-18 & 64,40 & Oct 2-18 \\
\hline & Baygorria & $88,18 \pm 0,33$ & 203,10 & Nov 2-18 & 65,50 & Oct 2-18 \\
\hline & Constitución & $90,64 \pm 0,14$ & 113,90 & Jun 2-18 & 76,00 & Nov 1-18 \\
\hline \multirow{3}{*}{$\begin{array}{c}\mathrm{SST}^{-1} \\
\mathrm{mgL}^{-1}\end{array}$} & Rincón del Bonete & $7,39 \pm 0,49$ & 16,60 & May 2-18 & 3,38 & Ene 2-19 \\
\hline & Baygorria & $9,92 \pm 0,41$ & 18,80 & Feb 1-18 & 1,60 & Feb 1-19 \\
\hline & Constitución & $8,23 \pm 0,40$ & 16,22 & Ago 2-18 & 2,67 & Feb 1-19 \\
\hline \multirow{3}{*}{$\begin{array}{c}\mathrm{MO}^{-1} \\
\mathrm{mgL}^{-1}\end{array}$} & Rincón del Bonete & $1,76 \pm 0,56$ & 5,00 & Abr 2-18 & 0,80 & Oct 2-18 \\
\hline & Baygorria & $1,88 \pm 0,39$ & 3,71 & Dic 2-18 & 0,80 & Feb 1-19 \\
\hline & Constitución & $1,56 \pm 0,27$ & 2,70 & Mar 1-18 & 1,10 & Oct $1-18$ \\
\hline \multirow{3}{*}{$\% \mathrm{MO}$} & Rincón del Bonete & $25,66 \pm 0,39$ & 46,00 & Dic 2-18 & 10,53 & Set $1-18$ \\
\hline & Baygorria & $21,35 \pm 0,47$ & 50,00 & Feb 1-19 & 11,28 & Jun 2-18 \\
\hline & Constitución & $21,75 \pm 0,45$ & 47,22 & Ene 1-19 & 9,70 & Jul 2-18 \\
\hline
\end{tabular}


En los tres sistemas de refrigeración (SR), la variación anual de la densidad de larvas de $L$. fortunei mostró comportamientos similares (Figura 3). Por otro lado, se observa una importante variabilidad en cada uno de los tres sistemas de refrigeración, reflejado en los desvíos estándar. Esta variabilidad podría estar explicada por la hidrodinámica en los diferentes sistemas de refrigeración de las tres $\mathrm{CH}$ (caudal, velocidad, tiempo de residencia, número de condensadores).

Las máximas densidades larvales se encontraron al inicio de la primavera y mediados del verano, con valores mínimos durante invierno. La $\mathrm{CH}$ Constitución presentó la densidad máxima (11603ind. $\mathrm{m}^{-3}$ ) durante febrero de 2019, donde la densidad larval promedio superó los 6000ind. $\mathrm{m}^{-3}$ a partir de fines octubre, manteniéndose hasta febrero. La CH Baygorria superó los 1000ind. $\mathrm{m}^{-3}$, mientras que Rincón del Bonete no alcanzó los 500ind. $\mathrm{m}^{-3}$.

La temperatura promedio de los tres SR mostró una correlación significativa positiva con el promedio de larvas $(r=0,63 ; n=54 ; p<0.001)$. Por otro lado, el incremento de la densidad larval en las tres $\mathrm{CH}$ se observó a partir de la segunda quincena de octubre 2018, cuando la temperatura del agua de los SR es mayor a $18^{\circ} \mathrm{C}$ (Figuras 2 y 3). La temperatura del agua es uno de los factores ambientales asociados al ciclo larval de $L$. fortunei, según diversos autores (Damborenea y Penchaszadeh, 2006; Darrigran, et al., 2007; Boltovskoy, et al., 2013; Giglio, et al., 2016). Dichos autores relacionan la presencia de larvas de la especie con temperaturas superiores a los $18^{\circ} \mathrm{C}$, coincidiendo con los resultados encontrados en el presente estudio.

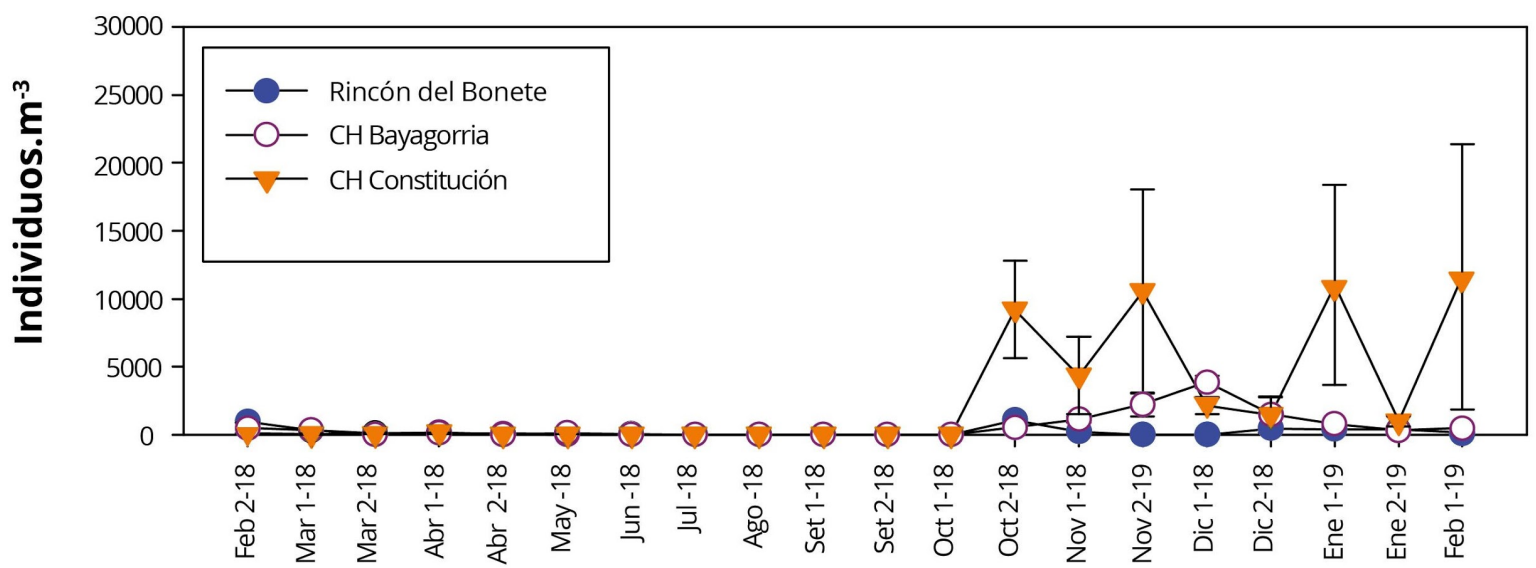

Fechas de muestreo

Figura 3. Variación de la denisdad promedio ( $\pm \mathrm{ds}$ ) larval de Limnoperna fortunei en las tres CH durante 2018-2019. 
En las tres $\mathrm{CH}$ se observó un incremento en la densidad larval a partir de la segunda quincena de octubre de 2018, que se corresponde con temperaturas del agua mayores a $18^{\circ} \mathrm{C}$ (Figuras 3 y 4). Los resultados coinciden con Brugnoli y otros (2011) en estudios realizados en el embalse Palmar (Río Negro). En los mismos se encontró un comportamiento estacional de larvas de $L$. fortunei con máximos durante la primavera y los inicios del otoño, y ausencia durante invierno, sugiriendo una reproducción contínua de la especie entre agosto y abril. Los resultados del presente trabajo sugieren la presencia de un descanso reproductivo de la especie durante épocas de temperatura del agua menor a $18^{\circ} \mathrm{C}$, en los embalses del Río Negro.

Por otro lado, las $\mathrm{CH}$ presentaron diferencias en las densidades promedio de larvas (Constitución > Baygorria > Rincón del Bonete), con hasta un orden de magnitud mayor en la $\mathrm{CH}$ Constitución durante los máximos larvales (Figuras 3 y 4). Estudios realizados en los embalses de las correspondientes $\mathrm{CH}$ evidenciaron que, durante el período 2000-2015, las biomasas de larvas de L. fortunei mostraron similares patrones que los observados en el presente trabajo. El embalse Palmar posee la mayor biomasa de larvas, seguido por Baygorria y Rincón del Bonete (Chalar, et al., 2010 y 2015). Este comportamiento podría asociarse a las características particulares de los embalses (por ejemplo, caudal, tiempos de residencia, disponibilidad de alimento, concentración de sólidos suspendidos totales o disponibilidad de sustratos consolidados), a las abundancias de organismos bentónicos, o al comportamiento biológico de las poblaciones del mejillón dorado (por ejemplo, potencial biótico, disponibilidad larval). Darrigran y otros (2007) afirman que, a pesar de que la temperatura es un factor condicionante, son necesarias otras variables para la liberación de larvas, como por ejemplo la disponibilidad de alimento. 


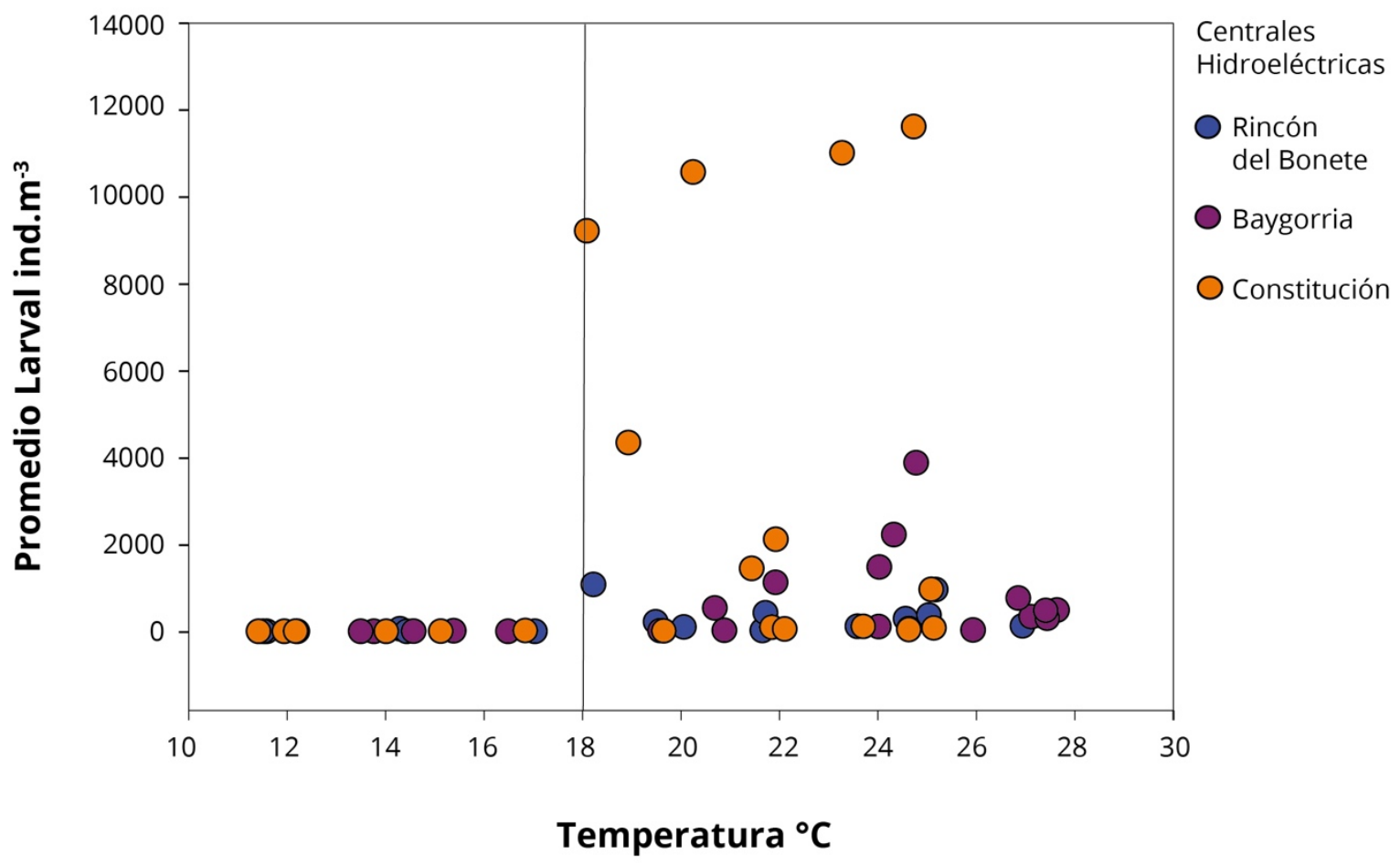

Figura 4. Temperatura del agua y densidad promedio larval de $L$. fortunei en las tres $\mathrm{CH}$ del Río Negro.

La densidad promedio de larvas no presentó correlaciones significativas con el $\mathrm{pH}$, SST y K; sin embargo, presentó asociaciones significativas con el \%MO $(r=0,34 ; n=54$; $p<0,05)$. El \%MO expresa la disponibilidad del alimento presente en el agua, siendo la fracción disponible para el consumo de adultos y larvas de $L$. fortunei. Las densidades larvales en la $\mathrm{CH}$ Baygorria presentaron un máximo (diciembre), mientras que en Constitución se identificaron cuatro máximos (octubre, noviembre, enero y febrero) (Figura 4). La CH Rincón del Bonete presentó promedios larvales bajos en comparación con los otros embalses, con un máximo en octubre. Chalar y otros (2011 y 2015) encontraron en estos embalses máximos larvales durante los mismos meses que el presente estudio. Estos máximos larvales están determinados por los ciclos gonadales de los individuos junto a otras características ambientales del sistema (por ejemplo, el tiempo de residencia de los embalses, las características de los SR de las diferentes $\mathrm{CH}$, la disponibilidad de oxígeno o el alimento) (Darrigran, et al., 2007, Giglio, et al., 2016; Ernandes-Silva, et al., 2017). La presencia de máximos de densidad larval en sitios donde la especie posee reproducción prolongada ha sido reportada para el alto Paraná (Brasil). En dicha zona, L. fortunei presenta reproducción durante todo el año, donde los máximos larvales se observaron entre diciembre y febrero, coincidiendo con épocas de mayor temperatura y disponibilidad de alimento (Ernandes-Silva, et al., 2017). Estos resultados concuerdan con los encontrados durante el presente estudio. 
El resultado del ACP (Figura 5) indicó que los dos primeros ejes explican el $62 \%$ de la varianza total de los datos. El primer factor explica el 39,4\% de la varianza y se puede asociar con los meses de mayor temperatura del agua, en donde las variables de mayor peso correspondieron a la temperatura, al promedio larval y al \%MO en agua, con la presencia de un patrón estacional. Por otro lado, el segundo componente explica el $22,4 \%$ de la varianza, asociado negativamente con $\mathrm{K}$, SST y $\mathrm{pH}$, aunque no se observa un patrón estacional claro con estas variables. Los meses que se relacionan positivamente con el primer eje corresponden a los meses de verano y primavera, caracterizado por presentar una mayor temperatura del agua y disponibilidad de alimento debido a las floraciones algales reportadas en el embalse (Chalar, et al., 2015). La temperatura y disponibilidad de alimento son factores asociados al aumento de las densidades larvales de L. fortunei en diferentes estudios (Darrigran, et al., 2007; Boltovskoy et al., 2009). En el presente estudio se considera el \%MO como un indicador de calidad del alimento, debido a que representa la fracción de los SST que pueden ser asimilados por la especie. El ACP (Figura 5) indica que el \%MO y los SST poseen una correlación negativa, lo que implicaría que el aumento de los SST se produciría por partículas inorgánicas, donde las densidades larvales estarían negativamente afectadas por los mismos. Las elevadas cargas de SST inorgánicos afectan a esta especie debido a que son organismos filtradores tanto en estadios larvales como adultos, ocasionando reducción en el área branquial en adultos; lo cual podría dificultar la obtención de alimento y oxígeno, además de aumentar la superficie de los palpos labiales, los cuales son órganos encargados de seleccionar el alimento. Debido a esto se perdería más energía seleccionando las partículas palatables (Paolucci, et al., 2014; Paolucci, 2021). Complementariamente, los SST reducen la penetración de la luz, afectando negativamente al fitoplancton como fuente de alimento para la especie, pudiendo generar reducción del oxígeno disuelto que se ha sugerido como una limitante para el establecimiento de la especie (Dodds y Whiles, 2010; Paolucci, 2021). Por lo anterior, los SST con bajo \%MO podrían disminuir la liberación de gametas o generar mortandad larval debido a que son alimentos de baja calidad. De esta manera, el ciclo larval estaría más condicionado por la temperatura del agua, y posiblemente por la calidad del alimento, que por el sitio de colecta $(\mathrm{CH})$. A pesar de estos resultados, existirían otros factores que podrían aportar a la dinámica larval y que no están siendo registrados en el ACP, como pueden ser las poblaciones de adultos en los embalses y sus afluentes, los caudales, el tipo de fitoplancton o la presencia de cianotoxinas (Boltovskoy, et al., 2009). 


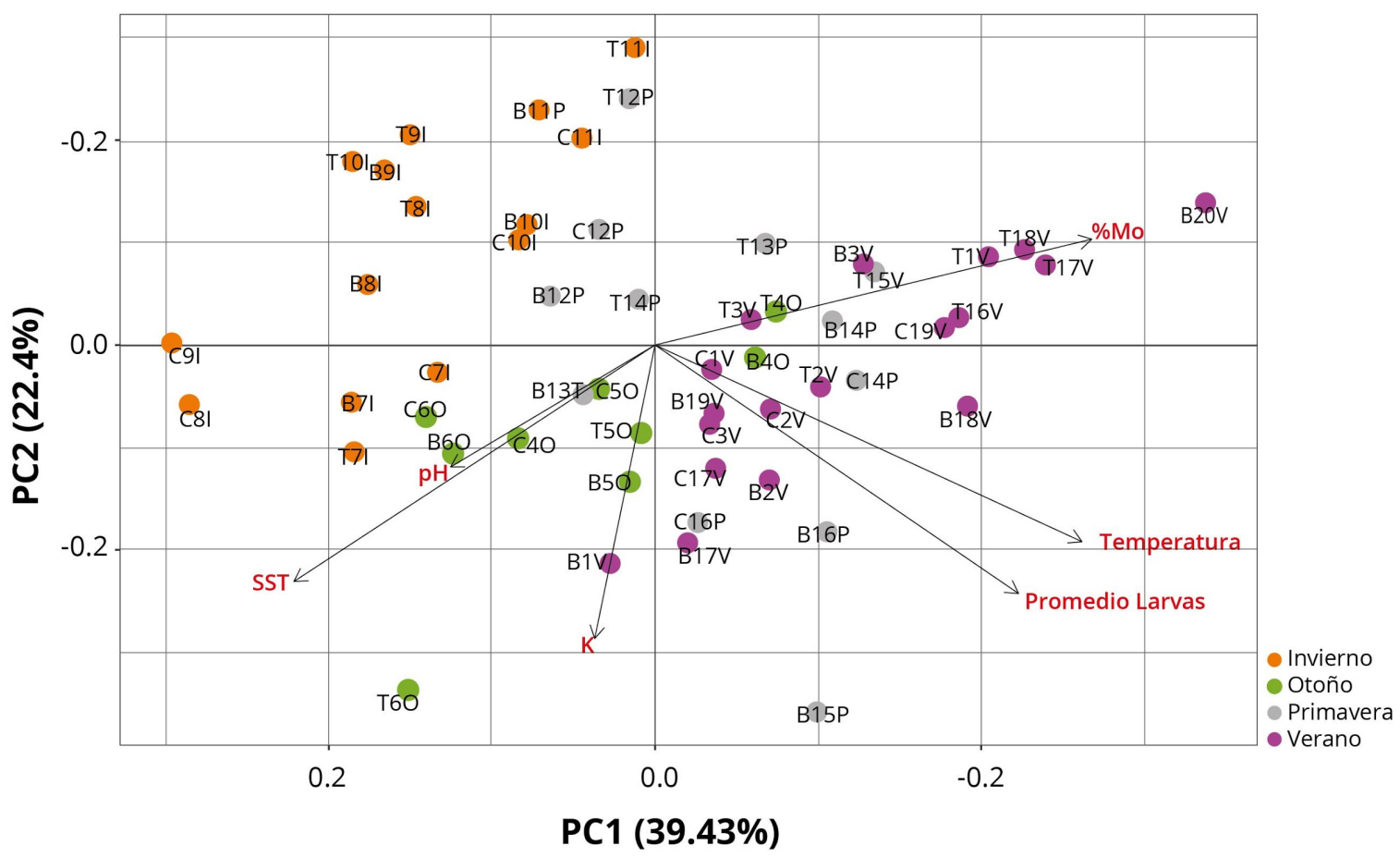

Figura 5. Diagrama de ordenación del Análisis de Componentes Principales para las tres $\mathrm{CH}$ durante 2018-2019. Variables ambientales y bióticas (flechas negras). $\mathrm{CH}$ : T: Rincón del Bonete, B: Baygorria, C: Constitución; número de muestreo (1-20) y estaciones del año: I: Invierno, O: Otoño, P: Primavera, V: Verano.

En otros sistemas acuáticos de la cuenca del Plata se ha sugerido que la aparición de $L$. fortunei estaría asociada al aumento de floraciones de cianobacterias potencialmente tóxicas del género Microcystis (Boltovskoy, et al., 2013). Dicha especie es capaz de filtrar selectivamente sobre el fitoplancton de pequeño tamaño y de poca movilidad, generando una disminución de la competencia para Microcystis spp. Esta especie de cianobacteria tiene la capacidad de formar colonias y regular su flotabilidad, no siendo fuertemente afectadas por la filtración del mejillón dorado (Gazulha, et al., 2012; Boltovskoy, et al., 2013; Silva y Giani, 2018). Boltovskoy y otros (2009 y 2013) describieron que altas concentraciones de Microcistinas L-R son capaces de matar las larvas de L. fortunei. Por otro lado, en los embalses del Río Negro, Chalar y otros (2015) observaron que durante los meses de verano ocurren las mayores concentraciones de toxinas, siendo este un factor que podría estar influyendo en las densidades larvales.

En los $\mathrm{SR}$ de las tres $\mathrm{CH}$, los estadios valvados dominantes fueron Velíger tipo $\mathrm{D}$ y Velíger preumbonada (Figura 6). Las densidades de los diferentes estadios larvales presentaron correlaciones con la temperatura del agua y el \% $\mathrm{MO}$, aunque con diferencias entre ellos (Tabla 3). 

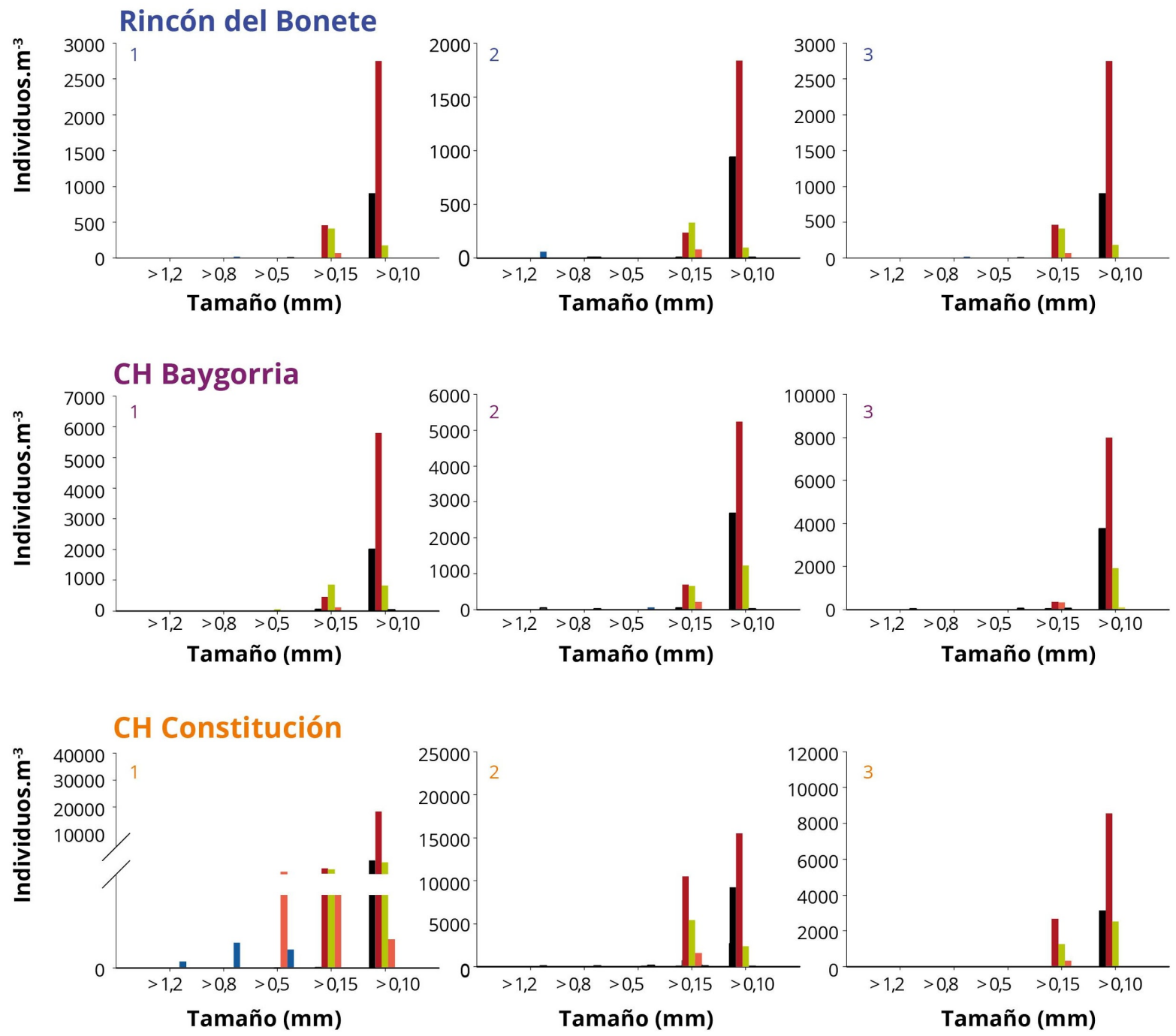

Velíger D

V. Preumbonada

V. Umbonada

Pedivelíger

Plantígrada

Figura 6. Densidad total (suma período febrero 2018-febrero 2019) de los estadios valvados de Limnoperna fortunei por tamaño en los sitios de muestreo (1-3) de los SR de las CH Rincón del Bonete, Baygorria y Constitución. 
Tabla 3. Correlaciones entre las densidades de los estadios larvales en las $\mathrm{CH}$ con temperatura del agua y porcentaje de materia orgánica. $R$ y $p$-valor ( ${ }^{*} 95 \%$ y ** 99\%). NS=No Significativo.

\begin{tabular}{|ccc|}
\hline Estadio Larval & Temperatura & $\%$ MO \\
\hline Velíger tipo D & $0,72^{\star *}$ & $0,34^{\star}$ \\
\hline Velíger preumbonada & $0,69^{\star *}$ & $0,34^{\star}$ \\
\hline Velíger umbonada & $0,47^{\star}$ & $0,28^{\star}$ \\
\hline Pediveliger & $0,38^{*}$ & 0,18 (NS) \\
\hline Plantigradas & $0,42^{\star}$ & 0,00 (NS) \\
\hline
\end{tabular}

El tamaño dominante de larvas correspondió a organismos mayores a $100 \mu m$, seguido de mayores a $150 \mu \mathrm{m}$. Los restantes tamaños $(500,800$ y $1200 \mu \mathrm{m})$ presentaron baja representación (Figura 6). El estadio de Velíger umbonada, Pedevelíger y Plantígrada (o Post-larvas) (tamaños que aproximadamente oscilan entre 200 y $800 \mu \mathrm{m}$ ) ocurrieron pocas veces y en pocos lugares (Figura 6). Sin embargo, en $\mathrm{CH}$ Constitución se registró una mayor cantidad de Plantígrada o Post-larva, principalmente en el sitio 1.

El primer estadio valvado de L. fortunei (Velíger tipo D) presenta tamaños entre 100 y $175 \mu \mathrm{m}$ (Cataldo, et al., 2005; dos Santos, et al., 2005). El tamaño de mallas consideradas en el presente trabajo $(100 \mu \mathrm{m})$ pudo haber generado un sesgo en las densidades larvales determinadas debido a una posible pérdida de individuos de menor tamaño de este estadio. A pesar de estas posibles limitaciones metodológicas, las mayores densidades de larvas correspondieron al estadio Velíger tipo D. Estas larvas provienen de los embalses de las mismas $\mathrm{CH}$, debido a que por los tiempos de residencia y la temperatura del agua no poseerían el tiempo necesario para alcanzar estadios más avanzados (Cataldo, 2015).

Los estadios más avanzados (Velíger preumbonada y Velíger umbonada) provienen de aguas arriba, posiblemente de los otros embalses, en el caso de Baygorria y Constitución. Sin embargo, en los tramos de río entre los mismos se genera una mortandad natural de las larvas, siendo que se ha registrado entre un 80 y $90 \%$ de mortandad de larvas entre el estadio Velíger preumbonada y Velíger umbonada en estudios de laboratorio y por depredación (Boltovskoy y Correa 2015; Cataldo, 2015). Adicionalmente, a medida que avanzan en su desarrollo comienzan a asentarse, haciendo que estén menos presentes en la columna de agua (Cataldo, 2015). Las altas mortandades previas del estadio Velíger umbonada explican por qué existe un declive 
tan drástico entre estos dos estadios. En Constitución se reportaron las máximas densidades, los mayores tamaños y la mayor variedad de estadios. Los estadios de mayor tamaño (estadio Plantígrado o Post-larva) se encuentran cercanos a su fase de asentamiento bentónico, siendo entonces el SR de la $\mathrm{CH}$ Constitución el más expuesto al asentamiento del mejillón dorado.

\section{CONCLUSIONES}

En los tres sistemas de refrigeración analizados, la densidad promedio y la densidad de los diferentes estadios larvales del mejillón dorado presentaron asociaciones con la temperatura del agua y el porcentaje de materia orgánica. Ambas variables han sido consideradas en otros estudios como variables que promueven la presencia de estadios larvales en sistemas naturales o artificiales invadidos por $L$. fortunei.

En los tres sistemas, a valores menores de $18^{\circ} \mathrm{C}$ en la temperatura del agua, la especie mostró un descanso reproductivo; y a valores superiores, el inicio del ciclo larval. El porcentaje de materia orgánica estaría relacionado con la disponibilidad de alimento en el sistema de embalses. En futuros estudios, esta variable debería considerarse un parámetro a analizar como promotora de un incremento en las densidades larvales, así como condicionante en la supervivencia de larvas y adultos.

Los estádios Velíger tipo D y Velíger preumbonada fueron los dominantes en las tres centrales hidroeléctricas. El tamaño dominante fue mayor a $100 \mu \mathrm{m}$, seguido por tamaños mayores a $150 \mu \mathrm{m}$, donde los estadios valvados de Post-larva se registraron principalmente en la $\mathrm{CH}$ Constitución, siendo el más expuesto al asentamiento y posterior macrofouling. Para lograr una mejor aproximación a la dinámica larval de los estadios larvales valvados de L.fortunei se recomienda utilizar para su colecta tamaños de malla más pequeños $(<100 \mu \mathrm{m})$; realizar monitoreos con una mayor periodicidad de colecta (semanal o diaria) principalmente durante épocas de mayor temperatura del agua, considerando simultáneamente sistemas hidráulicos (por ejemplo, sistemas de refrigeración) y ecosistemas acuáticos (por ejemplo, embalses, ríos).

En relación con el manejo, de acuerdo con el ciclo anual larval del mejillón dorado observado, la aplicación de métodos o estrategias de control en estos sistemas hidráulicos de las $\mathrm{CH}$ del Río Negro deberían focalizarse durante meses con mayor temperatura del agua y disponibilidad de alimento. Durante la primavera (setiembreoctubre) y el verano, las acciones de manejo deberían presentar la máxima intensidad, disminuyendo durante el otoño (a partir de mayo) y cesando en invierno (junioagosto).

Se recomienda continuar los estudios poblacionales de $L$. fortunei considerando estadios larvales (no valvados y valvados), así como estadios bentónicos en sistemas artificiales $(\mathrm{CH})$ y embalses en simultáneo. Estos estudios profundizarán la generación 
de conocimiento para la implementación de medidas de control y mitigación de la especie.

\section{AGRADECIMIENTOS}

El presente trabajo fue generado en el marco del Programa de Vinculación Universidad-Sociedad y Producción, CSIC-Udelar, Convenio Facultad de Ciencias- UTE "Limnoperna fortunei (mejillón dorado) en las presas y embalses del Río Negro, grado de invasión y aplicación de métodos de control mecánico". Fue presentado en el Simposio de "Ecología y Manejo del molusco invasor Limnoperna fortunei (mejillón dorado) en Uruguay", realizado en diciembre 2019 en Montevideo. Se agradece a la empresa UTE por su financiamiento y por el apoyo del personal, especialmente a la Gerencia de Planificación del Abastecimiento: Ing. Daniel Tasende, Cra. María del Rosario López, Cra. Leticia Severi, Ing. Alfonso Ruiz. En la Gerencia de Generación Hidráulica (Montevideo), Ing. Federico Sosa, así como en las Centrales Hidroeléctricas; $\mathrm{CH}$ Constitución: Ing. Gustavo Areco, Ing. Juan Deferrari, Alejandro Montaño, Kidia Perez, Gustavo Larrosa, Andrés Calvo, Luis Pombo, Luciano Alderete, David Ruiz, María Eugenia González; CH Baygorria: Ing. José Pirez, Atilio J. Brian Tejo, Carlos Pérez y Mario Otegui Báez (Empresa Estilo) y CH Rincón del Bonete: Ing. Pablo Thomasset, Ing. Alvaro Bellini, Fabian Cornalino, Miguel Tito, Luan Alvez y Luis Miguel Rodríguez. Muniz y Brugnoli agradecen a ANII-SNI. Los autores agradecen a los dos revisores que permitieron una mejoría en el documento final.

\section{REFERENCIAS}

Arocena, R., 2016. Sedimentos. En: Arocena, R., ed. Principios y métodos de limnología: ejemplos de Uruguay. Montevideo: DIRAC, Facultad de Ciencias. pp.59-72.

Boltovskoy, D., ed., 2015. Limnoperna fortunei. The ecology, distribution and control of a swiftly spreading invasive fouling mussel. Berlín: Springer Nature. DOI: 10.1007/978-3-319-13494-9

Boltovskoy D. y Correa, N., 2015. Ecosystem impacts of the invasive bivalve Limnoperna fortunei in South America. En: Hydrobiologia, 746, pp.81-95. DOI: https://doi.org/10.1007/s10750-014-1882-9

Boltovskoy, D., Correa, N., Bordet, F., Leites, V. y Cataldo, D., 2013. Toxic Microcystis (cyanobacteria) inhibit recruitment of the bloom-enhancing invasive bivalve Limnoperna fortunei. En: Freshwater Biology, 58, pp.1968-1981. 
Boltovskoy, D., Sylvester, F., Otaegui, A., Leites, V. y Cataldo, D., 2009. Environmental modulation of reproductive activity of the invasive mussel Limnoperna fortunei: implications for antifouling strategies. En: Austral Ecology, 34, pp. 719-730. DOI: https://doi.org/10.1111/j.1442-9993.2009.01974.x

Brugnoli, E., Clemente, J., Boccardi, L., Borthagaray, A. y Scarabino, F., 2005. Golden mussel Limnoperna fortunei (Bivalvia: Mytilidae) distribution in the main hydrographical basins of Uruguay: update and predictions. En: Anais da Academia Brasileira de Ciências, 77, pp.235-244. DOI: 10.1590/s000137652005000200004

Brugnoli, E., Clemente, J.M., Riestra, G., Boccardi, L. y Borthagaray, A. 2006. Especies acuáticas exóticas en Uruguay: situación, problemática y gestión. En: Menafra, R., Rodríguez, L., Scarabino, F. y Conde, D., eds. Bases para la conservación y manejo de la costa uruguaya. Montevideo: Vida Silvestre. pp.351-362.

Brugnoli, E., Dabezies, M., Clemente J.M. y Muniz, P., 2011. Limnoperna fortunei (Dunker 1857) en el sistema de embalses del Río Negro, Uruguay. En: Oecologia Australis, 15(3), pp.576-592.

Cataldo, D., 2015. Larval development of Limnoperna fortunei. En: Boltovskoy, D., eds. Limnoperna fortunei: the ecology, distribution and control of a swiftly spreading invasive fouling mussel. Berlín: Springer International Publishing. DOI: https://doi.org/10.1007/978-3-319-13494-9

Cataldo, D., Boltovskoy, D., Hermosa, J.L. y Canzi, C., 2005. Temperature-dependent rates of larval development in Limnoperna fortunei (bivalvia: mytilidae). En: Journal of Molluscan Studies, 71(1), pp.41-46. DOI: https://doi.org/10.1093/mollus/eyi005

Cataldo, D. y Boltovskoy, D., 2000. Yearly reproductive activity of Limnoperna fortunei (Bivalvia) as inferred from the occurrence of its larvae in the plankton of the lower Parana river and the Rio de la Plata estuary (Argentina). En: Aquatic Ecology, 34, pp.307-317. DOI: https://doi.org/10.1023/A:1009983920942

Chalar, G., Arocena, R., Pacheco, J.P. y Fabián, D.,2011. Trophic assessment of streams in Uruguay: atrophic state index for benthic invertebrates (TSI-BI). En: Ecological Indicators, 11, pp.362-369. DOI: 10.1016/j.ecolind.2010.06.004

Chalar, G., Fabián, D., González-Piana, M. y Delbene, L, 2010. Limnología de los embalses del Río Negro: noviembre 2000 - marzo 2009. Montevideo: Facultad de Ciencias, Sección Limnología. 
Chalar, G., Fabián, D., González-Piana, M. y Delbene, L., 2012. Informe final.

Caracterización limnológica de los embalses del Río Negro durante el período Setiembre 2009- Junio2011. Convenio UTE-Facultad de Ciencias [En línea]. Montevideo: Facultad de Ciencias, Sección Limnología. [Consulta: 05 de setiembre de 2020]. Disponible en:

http://limno.fcien.edu.uy/pactuales/Informe\%20Rio\%20Negro\%2020092011.pdf

Chalar, G., Fabián, D., González-Piana, M. y Piccardo, A., 2015. Estado y evolución de la calidad de agua de los tres embalses delRío Negro (período 2011-2015). Montevideo: UTE, Facultad de Ciencias, Sección Limnología.

Clemente, J.M.y Brugnoli, E., 2002. Record of Limnoperna fortunei (Dunker, 1857) (Bivalvia: Mytilidae) in continental waters of Uruguay (Río Negro and ríoYí). En: Boletín de la Sociedad Zoológica del Uruguay, 13, pp.29-33.

Clemente, J.M., Iglesias, C., Kröger, A., Lagomarsino, J.J, Méndez, G., Marroni, S. y Mazzeo, N., 2015. First record of the golden mussel Limnoperna fortunei Dunker, 1857 (Bivalvia: Mytilidae) in a lentic system in Uruguay. En: PanAmerican Journal of Aquatic Sciences, 10, pp.89-93.

Conde, D. y Sommaruga, R., 1999. A review of the state of Limnology in Uruguay. En: Wetzel, R. y Gopal, B., eds. Limnology in developing countries 2. Nueva Delhi: International Scientific Publications/SIL. pp.1-31.

Damborenea, C. y Penchaszadeh, P., 2006. Biología reproductiva de Limnoperna fortunei. En: Darrigran, G. y Damborenea, C., eds. Bio-invasión del mejillón dorado en el continente americano. La Plata: Universidad Nacional de La Plata. pp.69-82.

Darrigran, G., 2002. Potential impact of filter-feeding invaders on temperate inland freshwater environments. En: Biological Invasion, 4, pp.145-156.

Darrigran, G. y Damborenea, M.C., 2005. A bioinvasion history in South America. Limnoperna fortunei (Dunker, 1857), the golden mussel. En: American Malacological Bulletin, 20, pp.105-112.

Darrigran, G. y Ezcurra de Drago, I. 2000. Invasion of the exotic freshwater mussel Limnoperna fortunei (Dunker, 1857) (Bivalvia: Mytilidae) in South America. En: The Nautilus, 114, pp.69-73. 
Darrigran, G., Damborenea, C. y Greco, N., 2007. An evaluation pattern for antimacrofouling procedures: Limnoperna fortunei larvae study in a hydroelectric power plant in South America. En: AMBIO: A Journal of the Human Environment, 36(7), pp.575-579.

Darrigran, G., Martín, S.M., Gullo, B. y Armendáriz, L., 1998. Macroinvertebrates associated with Limnoperna fortunei (Dunker, 1857) (Bivalvia: Mytilidae) in Río de la Plata, Argentina. En: Hydrobiology, 367, pp.223-230.

Dos Santos, C.P., Würdig, N.L. y Mansur, M.C., 2005. Fases larvais do mexilhão dourado Limnoperna fortunei (Dunker) (Mollusca, Bivalvia, Mytilidae) na Bacia do Guaíba, Rio Grande do Sul, Brasil. En: Revista Brasileira de Zoologia, 22(3), pp.702-708.

Dodds, W. y Whiles, M., eds., 2010. Freshwater ecology: concepts and environmental applications of limnology. London: Elsevier. ISBN: 978-0-12-374724-2.

Ernandes-Silva, J., Pinha, G.D. y Mormul, R.P., 2017. Environmental variables driving the larval distribution of Limnoperna fortunei in the upper Paraná River floodplain, Brazil. En: Acta Limnologica Brasiliensia, 29, e108. DOI: https://doi.org/10.1590/s2179-975x7716

Ezcurra de Drago, I.,Montalto, I. y Oliveros, O., 2006. Desarrollo y ecología larval de Limnoperna fortunei. En: Darrigran, G. y Damborenea, C.,eds. Bio-invasión del mejillón dorado em el continente americano. La Plata: Universidad Nacional de la Plata, 220 pp.

Gazulha, V., Mansur, M.C.D., Cybis, L.F. y Azevedo, S.M.F.O., 2012. Grazing impacts of the invasive bivalve Limnoperna fortunei (Dunker, 1857) on single-celled, colonial and filamentous cyanobacteria. En: Brazilian Journal of Biology, 72(1), pp.33-39. DOI: http://dx.doi.org/10.1590/S1519-69842012000100004

Giglio, M.L., Mansur, M.C.D., Damboreanea, C., Penchaszadeh, P.E. y Darrigran G., 2016. Reproductive pattern of the aggressive invader Limnoperna fortunei (Bivalvia, Mytilidae) in South America. En: Invertebrate Reproduction \& Development, 1, pp.1-10. DOI: http://dx.doi.org/10.1080/07924259.2016.1174159

Haag, W.R. y Garton, D.W., 1992. Synchronous spawning in recently established population of the zebra mussel, Dreissena polymorpha, in western Lake Eire, USA. En: Hydrobiologia, 234, pp.103-110. 
Langone, J.A., 2005. Notas sobre el mejillón dorado Limnoperna fortunei (Dunker 1857) (Bivalvia, Mytilidae) em Uruguay. En: Publicación Extra. Museo Nacional de Historia Natural y Antropología, 1, pp.1-18.

Mansur, M.C., Pinhiero dos Santos, C., Darrigran, G., Hydrich, I., Calli, C. y Rossoni Cardoso, F., 2003. Primeiros dados quali-quantitativos do mexilhão dourado, Limnoperna fortunei (Dunker), no Delta do Jacuí, no Lago Gauíba e na Laguna dos Patos, Río Grande do Sul, Brasil e alguns aspectos de sua invasão no novo ambiente. En: Revista Brasilera Zoología, 20, pp.75-84.

Montalto, L., Oliveros, O., Ezcurra de Drago, I. y Denonte, L. 1999. Peces del río Paraná medio, predadores de una especie invasora Limnoperna fortunei (Bivalvia: Mytilidae). $F A B I C I B, 3(1)$, pp.85-101.

DOI: https://doi.org/10.14409/fabicib.v3i1.623

Muniz, P., Clemente, J. y Brugnoli, E.,2005. Benthic invasive pests in Uruguay: A new problem or an old one recently perceived? En: Marine Pollution Bulletin, 50, pp.993-1018.

Paolucci, E., Sardiña, P., Sylvester, F., Perepelizin, P., Zhan, A., Ghabooli, S., Cristescu, M., Oliveira, y MacIsaac, H., 2014. Morphological and genetic variability in an alien invasive mussel across anenvironmental gradient in South America. En: Limnology and Oceanography, 59(2), pp 400-412. DOI: https://doi.org/10.4319/lo.2014.59.2.0400

Paolucci, E., 2021. Ventajas y límites de la variabilidad morfofisiológica para el establecimiento, la colonizacióny la expansión del bivalvo invasor Limnoperna fortunei en Sudamérica. En: INNOTEC, 21, pp153 - 178.

DOI: https://doi.org/10.26461/21.09

Penchaszadeh, P., Darrigran, G., Angulo, C., Averbuj, A., Brigger, M., Dogliotti, A. y Pirez, N., 2000. Predation of the invasive freshwater mussel Limnoperna fortunei (Dunker, 1857) (Mytilidae) by the fish Leporinus obtusidens (Valenciennes, 1846) (Anastomidae) in the Río de la Plata, Argentina. En: Journal of Shellfish Research, 19, pp.229-231.

Ram, J.L., Fong, P.P. y Garton, D.W., 1996. Physiological aspects of zebra mussel reproduction: maturation, spawning, and fertilization. En: American Zoologist, 36, pp.326-338. 
Reavie, E.D., Jicha, T.M., Angradi, T.R., Bolgrien, D.W. y Hill, B.H., 2010. Algal assemblages for large river monitoring: Comparison among biovolume, absolute and relative abundance metrics. En: Ecological Indicators, 10, pp.167-177. DOI: https://doi.org/10.1016/j.ecolind.2009.04.009

Scarabino, F. y Verde, M., 1995. Limnoperna fortunei (Dunker 1857) en la costa uruguaya del Río de la Plata (Bivalvia; Mytilidae). En: Comunicaciones de la Sociedad Malacológica del Uruguay, 7, pp.374-375.

Silva, F.A. y Giani, A., 2018. Population dynamic of bloom-forming Microcystis aeruginosa in the presence of the invasive bivalve Limnoperna fortunei. En: Harmful Algae, 73,pp.148-156. DOI: https://doi.org/10.1016/j.hal.2018.02.007

Xu, H., Jiang, Y., Zhang, W., Zhu, M. y Al-Rasheid, K.A.S., 2011. An approach to determining potential surrogatesfor analyzing ecological patterns of planktonic ciliatecommunities in marine ecosystems. En: Environmental Science and Pollution Research, 18, pp.1433-1441.

DOI: https://doi.org/10.1007/s11356-011-0503-7 